\title{
Configurando Identidad de Aprendiz en la Escuela: Obstáculos en la Relación Pedagógica y su Incidencia en Procesos de “Deserción” Escolar Temprana
}

\author{
The Configuring of Apprentice Identity in School: Obstacles in \\ the Pedagogical Relation and its Incidence in Early School \\ Dropout Processes
}

\author{
Cristina Julio-Maturana * \\ Cooperativa Di-versos
}

\begin{abstract}
Considerando que la educación chilena busca avanzar en inclusión, el artículo amplia la comprensión de un problema vigente, la "deserción" escolar en contextos de pobreza, desde un enfoque identitario y de justicia simbólica. Sobre la base de una reflexión teórica sustentada en un estudio bibliográfico, se muestra que si los aprendices dejan inconclusas tempranamente sus trayectorias de aprendizaje escolar es porque en el inicio de las mismas se generaría un tipo de relación pedagógica que obstaculizaría la configuración de su identidad de aprendiz y la continuidad de sus aprendizajes escolares. Así, se da cuenta del macroconcepto de identidad de aprendiz, del de relación pedagógica, del enfoque de justicia simbólica y de los obstáculos para la configuración de la identidad de aprendiz en la escuela Finalmente se establecen los vínculos entre Identidad de Aprendiz, Relación Pedagógica y su incidencia en trayectorias de aprendizaje escolar, sustentando una comprensión cultural e intersubjetiva del problema.
\end{abstract}

Descriptores: Identidad, Aprendiz, Deserción escolar, Aprendizaje.

Considering that Chilean education is looking to move forward in inclusion. This article broadens the comprehension of a current issue: school desertion in poverty contexts, from an identity and symbolic justice approach. From a theoretical reflection based upon a bibliographic study, if learners leave early their school trajectory is because they generate, in the beginning of them, a pedagogical relationship that obstructs their learner's identity building and the continuity of their school education. Thus, this article portrays the macroconcept of learner's identity, pedagogical relationship, symbolic justice approach and the obstacles for the construction of learner's identity at school. Finally, the bonds between learner's identity, pedagogical relationship are shown, and their incidence in school learning trajectories, grounded from a cultural and intersubjective comprehension of the issue.

Keywords: Identity, Learner, School desertion, Learning.

*Contacto: cristina.julio.m@gmail.com

ISSN: 0718-7378

www.rinace.net/rlei/
Recibido: $\quad 15 / 11 / 2016$

$1^{\text {a }}$ Evaluación: 20/01/2017

Aceptado: 20/03/2017 


\section{Una aproximación al problema de la “deserción”}

Para iniciar la reflexión que propone el artículo cabe señalar dos cosas. La primera, que por "deserción" escolar se entiende el último eslabón de un proceso de "fracaso escolar" (García-Huidobro, 2001) que forma parte de un problema de des-igualdad/in-equidad que Chile viene presentando hace ya algún tiempo y cuyos niveles se han agudizado en los últimos años (OCDE, 2014). La segunda, la temporalidad en el proceso de "deserción" o "deserción temprana" refiere a que esta se genera en el primer ciclo de educación básica, es decir entre primero y cuarto básico, en el inicio del derrotero de los 13 años de escolaridad obligatoria en Chile. Por lo expuesto, el presente artículo ha optado por referir a trayectorias de aprendizaje escolar inconclusas como "deserción", toda vez que acentúa el aprendizaje como experiencia personal y continua, que ocurre antes y después de la escuela, enfatizando en el aprendizaje en el período de educación formal en la escuela o aprendizaje escolar.

Desde esta aproximación a la "deserción" escolar temprana y considerando gran parte de los estudios desarrollados y publicados en el país durante la década del 2000 hasta la fecha (Abraham y Lavín, 2008; Bellei y Fiabane, 2003; Boetto y Aracena, 2005; Castro y Rivas, 2006; Descouvieres, Redondo y Rojas, 2005; Espinoza et al., 2010, 2012, 2014; Goicovic, 2002; Ivanovic, 2000; JUNAEB, 2003; Marshall y Correa, 2001; Melis, 2001; Melis, Díaz y Palma, 2004; Méndez, 2002; Mertz y Uauy, 2002; Raczynski, 2002; Richards, 2009; Román, 2009; Schkolnik, 2003; Santos, 2009; Sapelli y Torche, 2004) es posible afirmar que las aproximaciones teóricas y metodológicas que ellos han asumido parecen insuficientes para abordar el problema de la "deserción escolar" temprana porque restringen el problema (Julio, 2014). Tal restricción a juicio de la autora deriva de dos cuestiones interrelacionadas. La primera, la primacía de un orden educativo (Tezanos, 2005, 2007) y la segunda, la primacía de un enfoque de in/justicia socioeconómica (Fraser, 2000, 2008). Coherentes con su objeto de estudio, la primera mira el problema desde la institución social y su materialización en el sistema escolar. Esta perspectiva, asociada a un predominio de injusticia socioeconómica, deja fuera del problema, la dimensión intersubjetiva del mismo. Precisamente, esta reflexión se hace cargo de esta dimensión inter-subjetiva con el fin de recuperarla e incorporarla en el análisis del problema. Este desafío de recuperación se hace más urgente en el contexto actual de la educación chilena, toda vez que se ha visto exigida por la Ley de No Discriminación $\mathrm{N}^{\circ}$ 20.609/2012, la Ley de Inclusión $\mathrm{N}^{\circ}$ 20.845/2015 y la Política de Convivencia escolar 2015-2018entre otras.

En efecto, los estudios parecen exiguos para asumir un problema de carácter complejo, sistémico, multifactorial e intersubjetivo que se va configurando desde el inicio de las trayectorias de aprendizaje escolar de los y las aprendices pertenecientes a familias en situación de pobreza. Por ello se opta por abordar el problema desde nuevos enfoques como: Una epistemología de la complejidad (Morin, 1999, 2002 y Morin, Roger y Motta, 2003), una perspectiva ecológica (Bronfenbrenner, 1987), una visión de justicia cultural o simbólica (Fraser, 2000, 2008), un paradigma sociocultural de aprendizaje (Baquero, 2006; Díaz, 2003; Julio, 2010, 2014; Lave, 1991; Lave y Wenger, 1991; Rogoff, 1993,

\footnotetext{
${ }^{1}$ Siguiendo a Goicovic (2002) se presenta "deserción” entre comilla porque este concepto pone el acento en un acto voluntario del niño, niña o joven, cuando se asume que más bien se trata de "actos de retiro escolar" generados mayoritariamente al interior del sistema educativo.
} 
1997; Wenger, 2001). En todos ellos asumiendo la cultura como concepto semiótico, como trama de significaciones a interpretar (Geertz, 2005). Enfoques en los que no es posible profundizar ahora por una cuestión de extensión pero que sin duda sustentan los planteamientos que se exponen.

Desde estos nuevos enfoques la identidad juega un papel relevante y genera una nueva comprensión del problema en contextos de pobreza. Desde ellos se expone la tesis de que si los aprendices dejan inconclusas tempranamente sus trayectorias de aprendizaje escolar es porque en el inicio de las mismas se generaría un tipo de relación pedagógica que actuaría como barrera u obstáculo en la configuración de su identidad de aprendiz, lo que dificultaría la continuidad de sus aprendizajes escolares. De este modo, a continuación se da cuenta del macroconcepto de identidad de aprendiz, del macroconcepto de relación pedagógica, de la dimensión de reconocimiento o la justicia cultural o simbólica, de los obstáculos en la relación pedagógica en la configuración de la identidad de aprendiz y la posibilidad de continuar trayectorias de aprendizaje escolar para finalmente establecer una relación entre identidad de aprendiz, relación pedagógica y trayectorias de aprendizaje, proponiendo una comprensión cultural e intersubjetiva del problema.

\section{Identidad de aprendiz: Un macroconcepto psicosocial, socioantropológico e interaccional}

La opción de asumir el problema desde un paradigma de la complejidad, permite pensar la identidad de aprendiz en la relación pedagógica como un macroconcepto, si entendemos por macroconcepto una forma de pensar desde el pensamiento complejo (Morin, Roger y Motta, 2003). Para construir este macroconcepto se toman elementos constitutivos del concepto de identidad y del concepto de aprendiz; no como una simple sumatoria de ellos, sino que se asume como un concepto cualitativamente sustantivo.

Para dar cuenta del macroconcepto se realiza una aproximación al concepto de identidad desde una perspectiva diacrónica y también sincrónica. Siguiendo a Gergen (1992), desde una perspectiva diacrónica es posible encontrar tres etapas históricoculturales en la concepción del yo y por ende en la concepción de identidad. Una primera etapa (siglo XIX) es en la que predomina una concepción Romántica del yo, en ella se atribuye a cada individuo rasgos de personalidad. En la segunda etapa (siglo $\mathrm{xx}$ ) predomina una concepción Moderna del yo, en esta se destaca como rasgo característico del yo, la capacidad de raciocino para el desarrollo consciente de la persona. En esta etapa nace la psicología como disciplina autónoma y se fortalecen las instituciones, en especial la educativa pues se cree que ellas pueden realizar un aporte al desarrollo consciente del sujeto a través de la razón. Una tercera etapa es la contemporánea, en la que predomina una concepción posmoderna del yo. En esta, se cree en la fragmentación del yo debido a la saturación social del sujeto que lo impulsa a realizar múltiples funciones y roles, proporcionándole una multiplicidad de lenguajes del yo, incoherentes y desvinculados entre sí. En esta etapa la posibilidad de identidad queda relegada de la mente del sujeto y sólo es posible un estado de construcción y reconstrucción permanente en el contexto del discurso social. La etapa actual del yo posmoderno que plantea Gergen desde la psicología social, es discutida por concepciones más convencionales de la psicología clínica, como las propuestas del yo posracionalista (Balbi, 2004). La diferencia 
fundamental entre ambas dice relación con la posibilidad de construir el sí mismo o identidad. Los posracionalistas aún creen en la posibilidad de construir una identidad única y en la posibilidad de hacerlo desde una situación endógena al sujeto. En definitiva, actualmente las corrientes provenientes de la psicología clínica convencional comparten la idea de que la identidad y el yo son un constructo. Sin embargo, la diferencia entre ellas estaría en el núcleo organizador del yo, la razón (Watzlawick, 1993 en Balbi, 2004) o las emociones (Bowlby, 1983; Guidano, 1999; Maturana y Varela, 1974,1984 citados en Balbi, 2004).

Desde una perspectiva sincrónica en cada etapa es posible reconocer la coexistencia de aproximaciones psicológicas, filosóficas y religiosas. Ellas dan cuenta de tradiciones y culturas diferentes respecto del yo, "algunas identifican un yo consciente y suelen describirlo en términos físicos; otras lo consideran una ilusión, y hay doctrinas religiosas que afirman que solo existe un yo firme y eterno al que suelen llamar Dios" (O'Brien y Guiney, 2003, p. 167). Nos quedamos con las aproximaciones psicológicas por su vinculación con temas de aprendizaje. Dentro de ellas también se encuentra una diversidad de visiones, desde las más convencionales, provenientes tanto de la psicología clínica como de la psicología social, hasta aquellas menos convencionales o más psicosociales e interaccionales (Íñigez, 2001).

Reconociendo la existencia de diversas corrientes que sustentan una concepción de identidad, la reflexión opta por un enfoque psicosocial (Íñiguez, 2001) y socioantropológico (Díaz, 2006; Giménez, 1997) y dentro de ellos, por una perspectiva interaccional. Es así como comprende que el macroconcepto de Identidad de aprendiz es una construcción individual/social que se va desarrollando sobre la base de una experiencia vital por ende subjetiva, interaccional y continua. Ciertamente, la identidad de aprendiz se construye en una relación interaccional que implica comunicación consigo mismo y con otros (Restrepo, 2007; Ysern, 2006) y la plena participación en comunidades de práctica (Wenger, 2001) porque desde un enfoque sociocultural de aprendizaje y sobre la base de los autores citados, así se aprende, participando en comunidades de práctica. Ciertamente, la identidad al ser una construcción permanente y continua en la vida, se va re-significando conforme la persona aprende y se desarrolla en procesos simultáneos, no lineales (Pérez-Taylor, 2002) de participación en diversas comunidades de práctica situadas, por lo que los otros inciden en su identidad individual/social y éste incide en la identidad de los otros. En estos procesos participativos que median en la configuración de la identidad de aprendiz influyen directamente los soportes identificatorios (Ainsenson 2002; Jorquera y Leiva, 2002), las interacciones, las mediaciones, negociaciones y también los artefactos culturales (Rogoff, 1993, 1997) propios de cada comunidad, entre ellos se destaca, la comunicación, el lenguaje y los vínculos afectivos. Todos estos elementos serán profundizados en el siguiente apartado referido a la relación pedagógica por su estrecha vinculación con ella.

Por lo expuesto, cabe afirmar que antes de iniciar el proceso de educación formal o escolarización, los niños y niñas (Da Costa, 2005, Gaitán, 2006; Unicef, 2006) ya se han convertido en genuinos aprendices porque han configurado una identidad de plenos y verdaderos aprendices en el seno de sus comunidades familiares. En sus cinco primeros años de vida han aprendido y se han desarrollado a través de su participación en la vida cotidiana de sus comunidades de práctica, en un entorno familiar y comunitario determinado, que en palabras de Rogoff (1993, 1997), son un verdadero currículo cultural, en tanto las comunidades familiares tienen metas y una organización propia 
para lograrlas. Es decir, los niños y niñas han aprendido qué y cómo se debe actuar en la comunidad a la que pertenecen. Con la ayuda de sus familiares y su entorno transitan desde la periferia de la comunidad o desde una participación periférica, hacia una plena participación (Lave, 1991) y se desenvuelven con autonomía y seguridad en sus comunidades gracias a la mediación de otros más experimentados, es decir han aprendido.

En efecto, en el microsistema (Bronfenbrenner, 1987) familiar los niños y niñas están en ambientes protegidos, ellos y ellas han conocido sus roles, conocen a quienes pueden apoyarlos/as en su autonomía, conocen las formas de convivencia, de relacionarse y comunicarse; se rigen por reglas y normas conocidas por ellos y ellas y saben reconocer donde está el poder del control social; saben cuál es su lugar en la familia, cómo negociar significaciones en ella y con quién/es debe hacerlo; conocen los artefactos que se utilizan y saben dónde encontrarlos, cómo y cuándo utilizarlos, en definitiva cada niño y niña, antes de entrar a la escuela, es un legítimo aprendiz. En estos primeros años de vida ha configurado sus aprendizajes culturales a través de la plena participación en y con su familia, cualquiera sea el tipo de ésta (Arteaga, Sepúlveda y Aranda 2012) y por lo mismo, también, ya tiene configurada su identidad personal/social como aprendiz.

Cuando los niños y las niñas inician el proceso de educación formal, inician un proceso de tránsito ecológico (Bronfenbrenner, 1987) en tanto se incorporan a un nuevo entorno llamado escuela, sin dejar de pertenecer y participar como legítimo aprendiz en el microsistema familia. Así entonces, ellos y ellas forman parte de un mesosistema configurado por la relación entre la familia, su comunidad y la escuela, o dicho en palabras de Rogoff (1993) en la relación entre el currículo cultural familiar y el currículo cultural escolar. Es allí, en la escuela, en la interacción con sus pares y figuras adultas (profesores y profesoras, paradocentes, auxiliares, etc.) donde comienza a re-significar su Identidad de aprendiz y asumir un nuevo rol. Sigue siendo niño o niña, continua con el rol de legítimo aprendiz en su familia, pero debe volver a construir, re-construir o reconfigurar su identidad de aprendiz en la escuela en tanto su conocimiento de este nuevo currículo escolar o el currículo cultural de la escuela, es aún desconocido para él o ella porque está comenzando a vivir la experiencia escolar. Según sea esta experiencia de tránsito en la nueva comunidad, más obstaculizadora o facilitadora de su participación en ella, será también la posibilidad de reconfigurar la identidad de aprendiz y por ende de continuar con su trayectoria de aprendizaje.

En definitiva, las escuelas materializan la educación formal de un sistema escolar concreto y como comunidad/es de práctica/s cultural/es específica/s, son un espacio de interacción, convivencia y comunicación interpersonal, donde la persona, en este caso los niños y niñas, construyen su identidad individual/social de aprendiz. Los entornos que circundan al aprendiz se amplían, el niño y/o la niña, junto a su familia y comunidad deben desarrollar estrategias identitarias (Kastersztein, 1999; Lipiansky, 1999; ToboadaLeonetti, 1999). Es decir, un conjunto de maniobras coordinadas para una victoria desde el principio de la dinámica de interacción, entendiendo que la "victoria de la identidad" es el propósito que las personas siguen cuando se trata de aceptar su actual estructura de identidad. Estas estrategias permiten a las personas mantener su mismisidad en un nuevo lugar, situado en un tiempo y un espacio. En este caso permiten a los niños y niñas mantener su identidad en la escuela. Es en este lugar llamado escuela donde se generarían algunos procesos que incidirían en la re-significación de la Identidad de Aprendiz. Precisamente los primeros años de escolaridad son centrales porque el ingreso 
a la escuela dice relación con una transición ecológica (Bronfenbrenner, 1987) que marcará su trayectoria vital como aprendiz. En este sentido no es un simple proceso de articulación curricular (Peralta, 2008) sino un profundo proceso de desarrollo identitario.

\title{
3. Relación pedagógica: Un macroconcepto que recupera inter-subjetividades
}

La relación pedagógica es definida según Tezanos (1983) como:

\begin{abstract}
una totalidad que expresa el contexto social en el cual se articula y se concreta a través del trabajo, la afectividad y el lenguaje como intermediaciones de la relación maestro-alumno que, a su vez, está centrada en un interés por la transmisión y apropiación de ciertos saberes aceptados como válidos por la sociedad en la cual está inserta. La relación pedagógica tiene como fin último el rompimiento de las relaciones de poder, a través del aprendizaje y consecuentemente del descubrimiento, por parte de los sujetos que en ella participan de las reglas de juego, las cuales definen los modos de relación en y con una determinada realidad social. (pp. 314315)
\end{abstract}

De este modo es posible asumir la relación pedagógica desde una perspectiva compleja, como un macroconcepto, una relación de relaciones, tal como lo muestra la figura 1. Ciertamente esta relación está construida por un objeto y un sujeto. El objeto es constituido por un supra-objeto compuesto, a su vez, por el saber social, el saber pedagógico y la mediación entre ambos. Asimismo, el sujeto se constituye en un suprasujeto compuesto por el maestro o profesor(a), el aprendiz o alumno(a) y los modos de relación entre ambos articulados por el trabajo, la afectividad y el lenguaje.

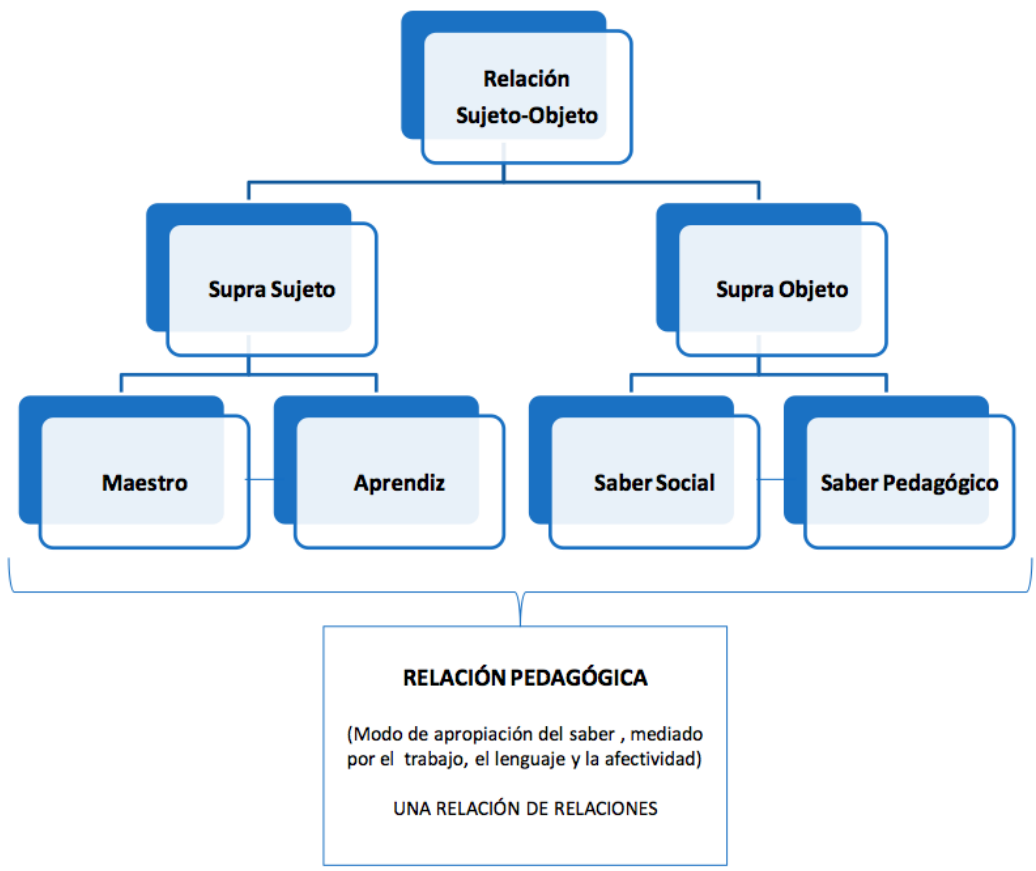

Figura 1. La relación pedagógica desde un discurso pedagógico sociocultural/transformador

Fuente: Recuperado de Julio (2014) basada en Tezanos (1983). 
El objeto en la relación pedagógica

es un objeto inmaterial del cual podemos hablar como un supra-objeto que está constituido por la relación que se establece entre el saber pedagógico y el saber social, los modos que adopta esta relación y las contradicciones que en ella surgen. (Tezanos, 1983 p. 317)

Siguiendo con la autora el saber social

está constituido por la acumulación de saberes "científico-filosóficos", saberes "comunes" y folclor que la sociedad ha construido a través de las transformaciones en las relaciones del hombre con la naturaleza y de las relaciones consigo mismo como sociedad. Pero el saber social no es cada uno de estos saberes, considerados separadamente, sino que está estructurado sobre la articulación, interacción y contradicción entre los mismos. El resultado de ellos es un "saber social hegemónico" que será transmitido institucionalmente a los sujetos sociales. (Tezanos, $1983 \mathrm{p}$. 318)

Asumiendo con la autora el saber hegemónico desde una perspectiva Gramsciana, este es dinámico y no estático, con contradicciones y fisuras, y no mecánico; porque, así como los grupos sociales generan su propia cultura, expresada en sus saberes, las relaciones sociales generan contradicciones y por ende también lo hacen las relaciones culturales, todo ello en la trama del devenir histórico de cada sociedad. Es así como en el contexto del discurso pedagógico, se levanta la pregunta sobre "cómo se median los modos de apropiación de este saber social hegemónico en la institución pedagógica" (Tezanos, 1983 p. 318). Esta mediación se realiza a través del saber pedagógico que "está constituido por los diferentes modos que adopta la mediación de la apropiación del saber social y se concreta en la práctica pedagógica institucional. Estos modos de apropiación expresan las visiones del mundo que se construyen en una determinada totalidad social, concretadas en el saber social hegemónico" (Tezanos, 1983 p. 318). Frecuentemente este saber pedagógico refiere al saber enseñar o, dicho de otra manera, a las formas de transmisión institucional del saber social, sustentadas en la reflexión entre la teoría y la práctica pedagógica concretadas a través de los métodos de enseñanza, por ello, es posible afirmar que "pertenece por derecho propio a los maestros, quienes lo elaboran, construyen y transforman” (Tezanos, 1983 p. 319).

Asimismo, el sujeto como un supra-sujeto está constituido por la relación que se establece entre maestros o profesores(as) y aprendices o alumnos (as) en el contex to de la institución educativa. Es una relación mediada por el trabajo, el lenguaje y la afectividad que permitirían o no, a los aprendices pasar desde una situación de heteronomía a una situación de autonomía. Es precisamente en esta institución "donde se concreta cotidianamente la relación pedagógica cuyas mediaciones articuladas tienen un fin último: el interés por la transmisión y la apropiación de saberes teóricos y prácticos" (Tezanos, 1983 p. 320). Dependiendo de los fines reproductivos (técnicos, mecanicistas e instrumentales) o de aquellos fines transformadores, la vivencia del trabajo, el lenguaje y los afectos puede asumir formas y características distintas en la institución educativa.

- El trabajo puede asumir dos formas, la alienante o la liberadora. Alienante toda vez que se sustenta en una concepción de trabajo alienado. Liberadora si se sustenta en una concepción de trabajo libre. Desde el enfoque marxista asumido por Tezanos (1983), la vivencia del trabajo pedagógico permitiría al ser humano apropiarse, organizar y transformar "la naturaleza y su relación consigo mismo y con los otros a través de la producción. El producir implica aquí actividad vital, genérica, libre y en consecuencia, 
creadora" (Tezanos, 1983 p. 320). Por el contrario, un trabajo alienado implicaría re-producir y la alienación de la persona en su producto "no sólo significa que el trabajo de éste se convierte en un objeto, en una experiencia exterior, sino además que su trabajo existe al margen de él, extraño a él y que se convierte en un poder autónomo frente a él, que la vida que le ha prestado al objeto se opone a él hostil y extraña" (Marx, 1844 en Tezanos, 1983 p. 320).

- El lenguaje en esta relación de relaciones se transforma en un medio de comunicación fundamental entre quienes interactúan, no sólo como un medio de relaciones interpersonales, sino también como un medio de apropiación del saber social a través del saber pedagógico propio de los profesores. Coherentes con el enfoque sociocultural de aprendizaje y semiótico de cultura, se asume el concepto de lenguaje de Halliday (1982) que aporta tres cuestiones a la reflexión. La primera, que los niños y las niñas aprenden o desarrollan potenciales significados que le ofrece su lengua materna como herramienta cultural, siempre en un contexto específico y en marcos normativos culturales "marco de regulación, de instrucción y de interacción personal de los padres y así por el estilo; y, recíprocamente, es "socializado" en los sistemas de valores y los modelos de conducta mediante el uso del lenguaje, al mismo tiempo que lo aprende" (Halliday, 1982 p. 35). Así, aprender el lenguaje "implica por una parte aprender a significar la lengua materna y al mismo tiempo aprender a usar el lenguaje como "instrumento de aprendizaje" (Halliday, 1982 p.32), por ello la preocupación del autor es el lenguaje natural en contextos reales de uso. La segunda, es que independiente de la lengua materna y de las culturas, hay algunas funciones del lenguaje que se cumplen en todas ellas, porque el niño y la niña a temprana edad se sienten motivados a desarrollar el lenguaje ya que descubren que este les facilita el cumplimiento de ciertos propósitos o cumple ciertas funciones para ellos. El autor propone tres metafunciones (experiencial o ideacional, interpersonal y textual) que no es el caso desarrollar aquí. Su tercer aporte es la ampliación de la teoría de los códigos propuesta por Bernstein (1988)², en tanto la reconoce, pero al mismo tiempo la discute desde una perspectiva cultural o semiótica, pues en ella lo relevante son las significaciones. Ciertamente afirma que aunque todos los seres humanos aprenden un sistema lingüístico en su contexto, al mismo tiempo:

los aspectos del sistema que se despliegan y se enfatizan de manera típica en uno $u$ otro tipo de uso son determinados en gran medida por la cultura, por los sistemas de relaciones sociales en que crece el niño, incluso los papeles que él mismo aprende a reconocer y a adoptar. Todos los niños tienen acceso al potencial de significado del sistema; pero pueden diferir, porque los grupos sociales difieren, en su interpretación de lo que exige la situación. (Halliday, 1982, p. 41)

\footnotetext{
${ }^{2}$ La teoría de los códigos refiere a las diferencias entre códigos elaborados y códigos restringidos que surgirían como consecuencia de las diferencias en la estructura social y de los tipos de relación social vinculados a ella, diferencias que generarían una falta de concordancia entre los órdenes simbólicos de significado de los estudiantes y aquellos de la escuela, obedeciendo a los distintos modelos de socialización de cada subcultura de una sociedad.
} 
- La afectividad se constituye en un tercer elemento de mediación. Esta se comprende de manera indisoluble con el intelecto, la cognición o la razón (Céspedes, 2008; Piaget, 2001) y como una construcción social y de significación cultural (Abramowski, 2010). Ciertamente se asume una concepción que supera la lógica binaria de "razón/pasión" propia de occidente porque ésta restringe a la persona si asume que el conocimiento humano comienza y termina en sí mismo y que las personas tienen como fin primero y último el conocimiento. Por el contrario, la afectividad "es el motor, la causa primera del acto de conocer; es el mecanismo que origina la acción y el pensamiento, lo cual implica afirmar que todo acto de deseo es un acto de conocimiento y viceversa" (Piaget, 2001, p. 8) y que está inscrita en "relaciones sociales y que su sentido se define dentro de determinados parámetros conceptuales y valorativos" (Abramowski, 2010, p. 34). Por ello, se entiende la afectividad como los sentimientos propiamente dichos y en particular las emociones y las pasiones, entendiendo que "cuando hablamos de afectividad (...) nos estamos refiriendo también a cuestiones más "intelectuales" como son los intereses, la simpatía y la antipatía por temas o personas, las actitudes de carácter ético, etc." (Piaget, 2001, p. 9) y que, por lo mismo, estos afectos, sentimientos, emociones y pasiones "no ocurren de modo natural, ni brotan de adentro hacia fuera, ni nacen del corazón y las 'entrañas"' (Abramowski, 2010, p. 33), sino que están inscritos en "relaciones sociales históricamente situadas, se construyen, se formatean, se regulan, se educan.” (Abramowski, 2010 p. 34). En efecto, según Céspedes (2008) todo niño y niña viene al mundo programado para la armonía y para ser feliz y para desarrollarlas y desarrollarse, cada cual necesita de la compañía de adultos que con amor le guíen y eduquen emocionalmente, entendiendo el amor como:

Una fuerza generativa inmensa e inagotable que está presente en cada ser humano antes de su nacimiento y se cultiva, pacientemente, a lo largo de las dos primeras décadas de la vida. Desde ese momento en adelante, el joven y luego el adulto poseerá en su interior un sólido bagaje amoroso que pondrá al servicio de su familia y de su comunidad. (Céspedes, 2008 p.13)

En el contexto de la institución educativa y del oficio de enseñar el profesor o la profesora tiene diversas maneras de querer (Abramowski, 2010). La autora las ha denominado afectos magisteriales, proponiendo a los profesores el desafío de "sacar los afectos de aquella zona recóndita y estrictamente singular para situarlos en un plano colectivo, social, cultural, histórico" (Abramowski, 2010 p.167) asumiendo que "cómo, cuánto, por qué y para qué los maestros querrán (o no) a sus alumnos será siempre, e inevitablemente, el resultado de lo que cada uno pueda hacer con "lo afectivo-pedagógico disponible de su época”. (Abramowski, 2010 p.168). Pero sin perder de vista que el o la maestra es uno de los adultos responsables de formar a sus alumnos para que alcancen su plena realización personal (Céspedes, 2008), es decir, tienen una gran responsabilidad social, más aún en contextos de Jornada escolar completa. 


\section{Justicia simbólica: El derecho al reconocimiento identitario}

La reflexión busca complementar y vincular dos dimensiones referidas a la deserción escolar temprana que comúnmente aparecen disociadas en los análisis de los fenómenos educativos y pedagógicos en la formación de niños y niñas provenientes de familias en situación de pobreza. Estas dimensiones son las de injusticia social o de redistribución y las de injusticia cultural o simbólica (Fraser, 2000), que en palabras de la autora no pueden estar disociadas porque "la justicia hoy día precisa de dos dimensiones: redistribución y reconocimiento" (Fraser, 2000 p.127). Sobre la injusticia social o socioeconómica tenemos mayor conocimiento, es la que más se ha trabajado para comprender los fenómenos de inequidad, desigualdad e injusticia social en educación y cuyas variables predominan en las investigaciones educativas en Chile ya antes señaladas. Para enriquecer el análisis del problema se incorpora en la reflexión el fenómeno desde la in/justicia cultural o simbólica, porque ella...

está arraigada en los modelos sociales de representación, interpretación y
comunicación. Ejemplos de la misma incluyen la dominación cultural (estar sujeto/a
a modelos de interpretación y que están asociados con una cultura ajena y son
extraños y/o hostiles a la propia); la falta de reconocimiento (estar expuesto a la
invisibilidad en virtud de las prácticas de representación, comunicación e
interpretación legitimadas por la propia cultura); y la falta de respeto (ser
difamado/a o despreciado/a de manera rutinaria por medio de estereotipos en las
representaciones culturales públicas y/o interacciones cotidianas). (Fraser, 2000
p.130)

Por lo expuesto es posible considerar en la relación pedagógica, la búsqueda de reconocimiento. Asimismo, es posible agregar que el reconocimiento de la identidad es un derecho humano de niños y niñas (Unicef, 2006). En el artículo 8 de la Convención sobre los Derechos del Niño se explicita este derecho y por lo mismo los estados han de ser garantes del mismo. La identidad representa el reconocimiento oficial de su existencia y de sus otros derechos. Por lo mismo, no basta el derecho a la educación si en la escuela no está garantizada su identidad. Es en este sentido que el artículo postula que los problemas educativos como el de la "deserción escolar", "fracaso escolar" o trayectorias de aprendizaje escolar inconclusas, no solo derivan de una inequidad/desigualdad o injusticia socioeconómica o de redistribución, sino fundamentalmente de una injusticia cultural o simbólica que invisibiliza en la escuela a los niños y niñas como legítimos aprendices (Julio, 2010) y en ellos, la identidad de "pobre", se superpone a la de aprendiz.

\section{Una relación pedagógica obstaculizadora para configurar identidad de aprendiz}

Sobre la base de los planteamientos entregados podemos adentrarnos en los obstáculos de la relación pedagógica que podrían dificultar la re-construcción o re-configuración de la identidad de aprendiz en la escuela, en contextos de pobreza y que a su vez incidirían en la posibilidad de continuar aprendizajes en ella. Si volvemos a Tezanos (1983) se puede afirmar que dependiendo del interés que determine y delimite el proceso de producción de conocimiento al interior de la disciplina pedagógica, serán los significados que se asignen a cada uno de los elementos que constituyen la relación pedagógica y los 
modos en que asuman sus relaciones. En este sentido existirían dos intereses, los reproductivos y los transformadores. Este artículo se sustenta en un interés transformador por lo que asume la relación pedagógica, desde una perspectiva críticoreflexiva dado que permite recuperar las relaciones intersubjetivas en el abordaje del problema.

Referimos a recuperar las relaciones intersubjetivas en tanto reconocemos como primer obstáculo que la educación chilena ha sido presa de una racionalidad instrumental que ha dado supremacía a modelos tecnológicos (Casassus, 2003, 2010; Cornejo, 2006; Cornejo y Redondo, 2007). Es decir, el primer obstáculo es macrosistémico, en tanto los modelos tecnológicos han dificultado los procesos de reflexión al interior del discurso pedagógico pues ha prevalecido una demanda mecanicista de la relación sujeto-objeto en el discurso pedagógico tradicional. En esta relación mecánica y tradicional

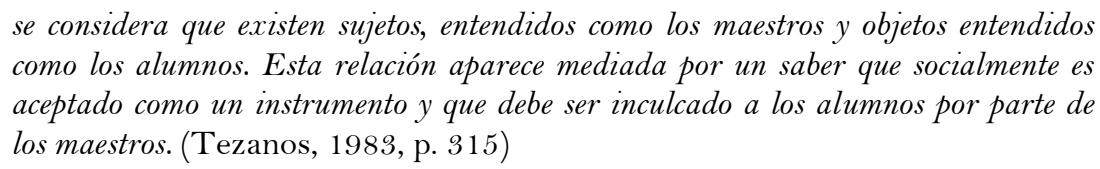
como los alumnos. Esta relación aparece mediada por un saber que socialmente es aceptado como un instrumento y que debe ser inculcado a los alumnos por parte de los maestros. (Tezanos, 1983, p. 315)

Asimismo, estos modelos imperantes han traído

$$
\begin{aligned}
& \text { como consecuencia el vaciamiento de sentido del término Pedagogía, limitando su } \\
& \text { significado a denotaciones inmediatas referidas sólo a las prácticas } \\
& \text { instrumentalizadas de los docentes, perdiendo ese término toda connotación con los } \\
& \text { procesos históricos, a lo largo de los cuales se construye el fundamento teórico que } \\
& \text { interactúa con esta práctica social. (Tezanos, 1983, p.314) }
\end{aligned}
$$

Tal como nos indica la misma autora en el artículo referenciado, las críticas a estos modelos han venido tanto desde las teorías reproductivistas, como la de los marxistas norteamericanos. Estas, aunque se sustentan en teorías diferentes coinciden en considerar que "la institución pedagógica es mediadora, reproductora y legitimadora del modelo social en el cual está inserta” (Tezanos, 1983 p. 316) y por ende determinaría el destino de cada individuo y sociedad. Sin embargo, desde estos modelos, la institución pedagógica nunca es vista como posible generadora de conflictos y transformación. Coherentes con una perspectiva compleja y sociocultural nos oponemos al determinismo de estas corrientes de pensamiento y adherimos con Tezanos (1983, 2007, 2012) a una propuesta transformadora a partir del discurso pedagógico materializado en la relación pedagógica. Es decir, desde un interés transformador se espera "recuperar la posibilidad de una reflexión al interior de la propia Pedagogía, que restablezca su carácter sociocultural y, por lo tanto, crítico" (Tezanos, 1983 p. 316)

La supremacía de esta racionalidad instrumental que se constituye en un obstáculo macrosistémico para la configuración de la identidad de aprendiz en la escuela, traspasa el sistema y llega al aula porque se materializa en modelos tecnológicos que inciden en cada uno de los diferentes constituyentes de la relación pedagógica. Dicho de otro modo, este obstáculo macrosistémico genera a su vez, obstáculos en la relación pedagógica, de orden microsistémico. En efecto, cada uno de los elementos constituyentes de la relación pedagógica se transforma en un obstáculo en sí mismo para la re-configuración identitaria de los aprendices, reflexión que desarrollamos a continuación.

En relación al supraobjeto, las tendencias cientificistas de la Pedagogía asumen el enseñar como 
sinónimo de inculcación acrítica del saber social, tendiendo a instrumentalizar al sujeto social (...) [y] el aprender deviene en un proceso memorístico y repetitivo del saber social que, en función de una racionalidad instrumentalizadora, impone representaciones y no permite un cuestionamiento sobre el fin de las mismas. (Tezanos, 1983 p. 316)

Por ello cabe preguntarse por la relación entre el saber social y el saber pedagógico, la mediación entre ellos "permite la posibilidad de la transformación dinámica de ambos saberes o el mantenimiento estático de los mismos" (Tezanos, 1983 p. 320). Las respuestas variarán según la aproximación que se asuma. Desde una aproximación cientificista de carácter instrumental, sustentada en la eficiencia (enseñar como inculcación y aprender como repetición) como la que predomina en Chile (Casassus, 2003, 2010; Cornejo, 2006; Cornejo y Redondo, 2007), se tendería al mantenimiento estático de ambos saberes. Esta eficiencia, según Tezanos (1983),

está dirigida al encubrimiento de las relaciones de poder que legitiman el sistema
social y está vinculada al trabajo que tiene como fin último la productividad y la
reproducción de la estructura económica y cultural en la cual está inserta. No existe
aquí la posibilidad de una contradicción entre ambos saberes, puesto que uno (el
pedagógico) se encuentra al servicio del otro (el social). (Tezanos, 1983 p. 316)

En este sentido, este saber pedagógico pierde autonomía y se transforma en saber dominado porque se supedita al social y se hace un saber homogéneo, sin fisuras ni contradicciones.

Desde esta misma perspectiva instrumental el énfasis en el suprasujeto estaría asociado a la adquisición de habilidades y destrezas disociada de saberes teóricos. Por ejemplo, en el primer ciclo de educación básica, los procesos de alfabetización en la lengua materna o bien, las operaciones básicas en matemática se enseñan con escasas posibilidades de preguntarse, tanto por parte de profesores y estudiantes, por el porqué de dichos saberes, las valoraciones culturales y sus tensiones. Es decir, se asumen como prescripciones curriculares o mandatos ministeriales incuestionables sin posibilidad de pertinencia cultural.

Al mismo tiempo, si se revisa cada uno de los elementos de la mediación (trabajo, lenguaje y afectos) desde la perspectiva instrumental ya señalada, es posible observar que estos tres elementos también se constituyen en obstáculos en sí mismos, a saber:

- El trabajo se transforma en un obstáculo de la configuración de la identidad de aprendiz si este es alienante, es decir si las formas metodológicas que son la concreción de los métodos de enseñanza en la institución educativa generan un método

donde la relación maestro-alumno está regida por un interés técnico y de control, donde los saberes y prácticas sociales que son mediadas pretenden aparecer como exteriores a ambos elementos de la relación y ser los dominantes en el contexto social donde está articulada la institución pedagógica. (Tezanos, 1983, p. 321)

- Desde esta perspectiva, los saberes son homogéneos sustentados en una base de "cientificidad" por lo que los contenidos pedagógicos dan cuenta de estos saberes desconociendo "los procesos históricos de su desarrollo y su articulación y contradicción con el sentido común y el folclore” (Tezanos, 1983, p. 321) de cada cultura. Es así como el trabajo del maestro(a) o del profesor(a) deviene en inculcar dichos saberes a sus alumnos al interior de la institución pedagógica porque "el objeto de este trabajo y su producto es 
ajeno tanto a uno como a otro elemento de la relación (...) y su contenido aparece como una parcialización más dentro de las estructuras programáticas de la institución (Tezanos, 1983 p. 322). Aunque el discurso pedagógico predominante en el sistema educativo nacional e internacional se sustenta en los intereses y necesidades de todos los alumnos y todas las alumnas "estos intereses y necesidades están significados desde el exterior de la institución, siendo impuesto a la misma como un resultado más para transmitir" (Tezanos, 1983 p. 322). y lo que es peor aún sin considerar la opinión, la experiencia, trayectorias y proyectos de vida de los sujetos involucrados en la relación maestro(a)/profesor(a)-aprendiz/alumno(a) que cada vez más, abandonan la institución educativa formal y lo hacen más tempranamente. Este modo de trabajo alienado legitimaría ciertos saberes y prácticas sociales útiles para un saber dominante determinado, que como ya fue dicho, es proclive a la reproducción del sistema social en la institución educativa, independiente de los tiempos y lugares donde esté situada. En definitiva, asumir el saber separado de los contextos y sus contradicciones hace que el trabajo pedagógico sea alienado, aunque tenga apariencia de libre desde el discurso teórico vigente. En efecto, sigue siendo alienado cuando referimos

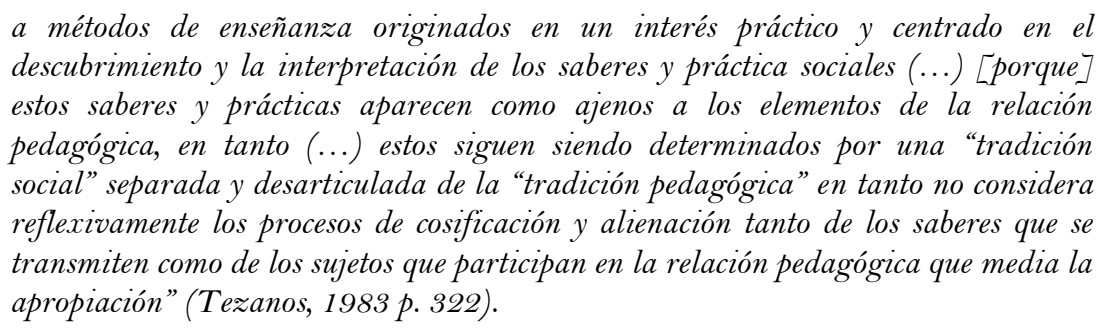

Desde esta concepción de trabajo alienado el papel del maestro(a) o profesor(a) se restringe al de inculcador y sus métodos tienden a devenir en modos de control, disciplinamiento, restringiendo y desvirtuando la relación maestro(a)/profesor(a)aprendiz/alumno(a).

- El lenguaje, asumido desde los tres aportes planteados por Halliday (1982), en la relación pedagógica con niños y niñas que provienen de contextos de pobreza, se puede transformar en un obstáculo si se sigue asumiendo restringidamente como una cuestión de códigos lingüísticos. En efecto, es posible afirmar que los encuentros/vínculos o desencuentros/desvinculaciones en la relación pedagógica en toda su complejidad, no son sólo un problema de códigos entre diferentes culturas, sino muy especialmente un problema de interpretaciones que devienen de las redes de significaciones de cada cultura, en este caso la cultura familiar de cada aprendiz y la cultura escolar. Si la relación pedagógica es una relación de relaciones el problema de las interpretaciones se hace aún más complejo, toda vez que supera las diferencias en las relaciones interpersonales propias del supra sujeto en tanto incluye las relaciones del supra objeto. Relaciones, las unas y las otras, mediadas tanto por el trabajo (libre o alienado) del que ya dimos cuenta y la afectividad, que revisaremos a continuación. 
- La afectividad o la vivencia de emociones y afectos en la relación pedagógica pueden constituirse en un obstáculo para la configuración de la identidad de aprendiz en los primeros años de escolaridad cuando se asumen de manera disociada de la cognición, toda vez que la afectividad es indisoluble con el intelecto (Céspedes, 2008) y es el motor del conocimiento (Piaget, 2001). Asimismo, se transforma en obstáculo cuando las diferentes emociones y sus expresiones sólo se interpretan desde la mirada adultocéntrica o desde la cultura escolar homogeinizante y no se conciben por parte del profesorado como una construcción social y de significación cultural en contextos situados (Abramowski, 2010). Sobre la base de los autores trabajados, la afectividad se transforma en un obstáculo cuando la entendemos disociadas de intereses, simpatía, antipatía por temas y personas, actitudes, etc. También se transforma en obstáculo si entendemos que los afectos y las emociones han de ser homogéneas, es decir que cada estudiante debe tener las mismas conductas y comportamientos en la escuela, y que las emociones que las sustentan deben ser los que esperan los adultos en la escuela, independiente de la condición y trayectoria de vida y de aprendizaje de cada aprendiz.

\section{Cerrando la reflexión. Una comprensión cultural e intersubjetiva del problema}

Para cerrar la reflexión propuesta, es preciso tener en cuenta dos cosas. La primera, que los niños y las niñas (Gaitán, 2006; Da Costa, 2005; Unicef, 2006), que provienen de familias en situación de pobreza y que se incorporan a la escuela básica han construido una legítima identidad de aprendiz a través de su participación en comunidades de práctica y a través de los currículos culturales familiares, con sus propias metas y modos de apropiación de los mismos (Rogoff, 1993, 1997). Por lo mismo, su conflicto no es sólo pertenecer a familias en condición de "pobreza" sino fundamentalmente por pertenecer a comunidades culturales familiares que tienen significaciones y modos de interpretar los saberes sociales y pedagógicos de manera diferente a las de la cultura escolar. Es decir, la trayectoria de aprendizaje escolar de estos legítimos aprendices está tensionada por la discrepancia de interpretaciones (Halliday, 1982) derivadas de la diversidad de currículos culturales familiares (Arteaga, Sepúlveda y Aranda, 2012) que chocan o discrepan que con el currículo cultural escolar tradicional (Julio, 2014) y las creencias y prácticas predominantes de profesores (Julio y otros, 2016). La segunda, que la perspectiva transformadora asumida en la presente reflexión, invita a re-visar por una parte, a la institución educativa para que florezca como re-creadora de los saberes sociales acumulados, porque permiten la articulación con la adquisición de las prácticas sociales situadas y por otra, a re-significar la relación pedagógica desde su complejidad y como posibilidad de encuentro intersubjetivo mediado por el lenguaje, el trabajo y los afectos.

Sobre la base de estas consideraciones la propuesta teórica desarrollada establece tres vínculos de reciprocidad entre la identidad de aprendiz, la relación pedagógica y las trayectorias de aprendizaje escolar como un nuevo modo de comprender el problema de la "deserción" escolar temprana en legítimos aprendices que provienen de familias en situación de pobreza. Tal como lo muestra la figura 2, estos vínculos refieren a los grados de participación en la relación pedagógica, los afectos o la vivencia afectiva en ella y las significaciones compartidas o significados culturales comunes en esta relación. 
Cada uno de los tres vínculos, al ser recíprocos, inciden directamente en el aprendizaje, la identidad de aprendiz y la continuidad de trayectorias, como se sustentará a continuación.

\begin{tabular}{|c|c|c|}
\hline \multicolumn{2}{|c|}{ Vinculaciones entre identidad de Aprendiz (IA); Relación Pedagógica (RP) y Trayectoria } \\
de Aprendizaje escolar (TAE)
\end{tabular}

Figura 2. Vínculos de reciprocidad entre identidad de aprendiz, relación pedagógica y trayectoria de aprendizaje escolar Fuente: Julio (2014).

Sustentados en el enfoque sociocultural de aprendizaje asumido, se aprende y se desarrolla "a través de la participación guiada en la actividad social con compañeros/as que apoyan y estimulan su comprensión y su destreza para utilizar los instrumentos de la cultura" (Rogoff, 1993, p. 21). Asimismo, desde este enfoque cada aprendiz configura y recorre su propia historia de vida escolar en el "incesante entrelazamiento" de dos procesos: el de participación (la propia experiencia de participación) y la cosificación (formas de artefactos de representación: instrumentos, conceptos, términos, etc.) (Wenger, 2001). En efecto, cada historia vital se constituye a través del recuerdo de la propia experiencia y su interpretación y reinterpretación en el proceso de interacción social, en una historia compartida y situada. En este sentido, los niños, en su condición de seres humanos, son legítimos aprendices porque cada cual -independiente de su condición socioeconómica- ha recorrido una/su trayectoria vital. En ella ha aprendido, se ha desarrollado y ha construido su identidad y lo ha realizado en el contexto de sus comunidades de práctica a través de un proceso personal de apropiación participativa del saber, generado por medio de un proceso interpersonal de participación guiada en un sistema de aprendizaje comunitario o institucional (Rogoff, 1997). Por ello es relevante en la construcción identitaria de aprendiz escolar, la historia de la participación de cada niño en las prácticas culturales (Rogoff y Gutiérrez, 2003) propias de la relación pedagógica en una escuela básica municipal.

La reciprocidad en el vínculo se establece en los grados de participación. Si los niños y niñas alcanzan una plena participación (Lave, 1991) en la relación pedagógica en la escuela, tendrán mayores posibilidades de aprender, desarrollarse y construir una identidad de aprendiz legitimada y con ello aumentan las posibilidades de concluir trayectorias de aprendizaje escolar. Por el contrario, si los niños se quedan en situaciones de participación periférica en la relación pedagógica en la escuela, las posibilidades de aprendizaje, desarrollo y construcción de la Identidad de aprendiz se ve 
limitada y con ello se restringe las posibilidades de concluir trayectorias de aprendizaje escolar.

Lo mismo que con la participación, la experiencia afectiva vivida en la relación pedagógica tiene una relación recíproca con la identidad de aprendiz y con la continuidad de trayectorias de aprendizaje escolar.

Considerando los planteamientos de Piaget (2001), Maturana (2007), Céspedes (2008), Abramowski (2010) y Milicic $(2001,2012)$ es posible afirmar que las emociones son el motor del conocimiento y por ende del desarrollo humano. Sólo gracias a la vivencia y experimentación de la emoción del amor, los niños pueden sentirse como legítimos otros en la relación pedagógica. Si esta experiencia no ocurre, las interacciones dejan de ser relaciones sociales, la convivencia se deteriora, los niños comienzan a "enfermarse o marchitarse", van alterando su desarrollo y van dejan de sentirse legitimados por los otros y también por sí mismos, en tanto persona y aprendiz. En el caso de la escuela dejan de sentirse legitimados por sus compañeros, pero muy especialmente por los adultos. De este modo sólo una convivencia afectiva amorosa en la relación pedagógica permite a los niños configurar una identidad de legítimo aprendiz y ella a su vez le permitiría continuar sus trayectorias de aprendizaje escolar. Si esta experiencia no es tal, la identidad de aprendiz se deslegitima y se pierde el sentido de continuar trayectorias de aprendizaje escolar.

Un tercer vínculo de reciprocidad entre Identidad de Aprendiz, Relación Pedagógica y trayectorias de aprendizaje escolar es la sintonía en los significados o significados compartidos que tenga el niño en el proceso pedagógico, especialmente en la relación pedagógica o los modos de apropiación del saber. Cuando referimos a significaciones damos cuenta de la proximidad cultural entre el currículo cultural familiar comunitario (Rogoff, 1997) y el currículum cultural escolar o formal (Magendzo, 2008); entendiendo por cultura la acepción semiótica otorgada por Geertz (2005) y ya desarrollada. Coherente con los planteamientos del autor que, siguiendo a Max Weber, reconoce que el ser humano "es un animal inserto en tramas de significaciones que él mismo ha tejido" (Geertz, 2005 p. 20), la cultura es precisamente esa urdimbre que es necesario interpretar. Por ello, si la red de significaciones que los niños construyen tempranamente en las relaciones pedagógicas que les ha tocado vivenciar en la escuela municipal, son más próximas a las significaciones de sus modos culturales infantiles populares, la relación pedagógica será interpretada como cercana y con sentido para ellos. Será una relación pedagógica que favorecerá la construcción de una identidad de aprendiz legitimada y con ello cobrará sentido la continuidad de trayectorias de aprendizaje escolar. Por el contrario, si las significaciones tienen mayores grados de diferencia, costará que se comprenda la cultura escolar y la relación pedagógica en ella; si esto ocurre la construcción de la Identidad de aprendiz se verá deslegitimada y con ella se perderá el sentido por continuar trayectorias de aprendizaje escolar en la escuela municipal.

De este modo podemos cerrar la reflexión preguntándonos ¿iSi todos los niños y niñas son legítimos aprendices por qué los que provienen de familias en situación de pobreza dejan inconclusas tempranamente sus trayectorias de aprendizaje escolar? La respuesta que proviene de la tradición de estudios educativos como los referidos al inicio de la reflexión, dan cuenta mayoritariamente de la multifactorialidad del fenómeno poniendo énfasis en aquellos aspectos estructurales y de in/justicia social o de redistribución en 
educación. Esta reflexión complementa la respuesta afirmando que el fenómeno o problema de la "deserción" escolar temprana o la continuidad de trayectorias de aprendizaje en la escuela dice relación con un fenómeno complejo, sistémico, de carácter pedagógico, interaccional, intersubjetivo y cultural, porque refiere a procesos de formación de personas que provienen de contextos históricos situados, por profesionales también personas, responsables de su formación. Por ende, la consideración de la justicia social y de redistribución parece insuficiente y se hace necesario la consideración de una justicia cultural o simbólica referida al reconocimiento de identidades diversas, en este caso de legítimo aprendiz.

\section{Referencias}

Abraham, M. y Lavín, S. (2008). La deserción escolar: un desafío pedagógico y social. Paulo Freire. Revista de Pedagogía Crítica, 7(6), 147-168.

Abramowski, A. (2010). Maneras de querer. Los afectos docentes en las relaciones pedagógicas. Buenos Aires: Paidos.

Aisenson, D. (2002). Después de la escuela. Transición, construcción de proyectos, trayectorias e identidad de los jóvenes. Buenos Aires: Editorial Universitaria de Buenos Aires.

Arteaga, C., Sepúlveda, S. y Aranda, V. (2012). Diversificación de las estructuras familiares: Caracterización de las convivencias en Chile. Revista de Sociología, 27, 37-52.

Balbi, J. (2004). La mente narrativa: Hacia una concepción posracionalista de la identidad personal. Buenos Aires: Paidós

Baquero, R. (2006). Sujetos y aprendizaje. Buenos Aires: Ministerio de Educación, Ciencia y Tecnología de la Nación.

Bellei, C. y Fiabane, F. (2003). 12 años de escolaridad obligatoria. Santiago: LOM Ediciones.

Boetto, C. y Aracena, M. (2005). Estudio Exploratorio sobre la Visión de Salud de un Grupo de Adolescentes Hombres Desertores del Sistema Escolar de la Comuna de Santiago, Chile: Sus Necesidades, Aspiraciones, Prioridades y Propuestas. PSTKHE, 14(2), 63-79. doi: $10.4067 /$ S0718-22282005000200006

Bronfenbrenner, U. (1987). La ecología del desarrollo humano. Barcelona: Paidós

Casassus, J. (2003). Escuela y la (des)-igualdad. Santiago: LOM.

Casassus, J. (2010). Las reformas basadas En estándares: Un camino equivocado. Educere et Educare, 5(9), 85-107

Castro, B. y Rivas, G. (2006). Estudio sobre el fenómeno de la deserción y la retención escolar en localidades de alto riesgo. Revista Sociedad Hoy, 2(11), 35-72.

Céspedes, A. (2008). Educar las emociones. Educar para la vida. Santiago: Vergara.

Cornejo, R. (2006). El experimento educativo chileno 20 años después: Una mirada crítica a los logros y falencias del sistema escolar. REICE. Revista Electrónica Iberoamericana sobre Calidad, Eficacia y Cambio en Educación, 4(1), 1-24.

Cornejo, R. y Redondo, J. M. (2007). Variables y factores asociados al aprendizaje escolar. Una discusión desde la investigación actual. Estudios Pedagógicos, 33(2), 155-175. doi: $10.4067 / \mathrm{s} 0718-07052007000200009$

Da Costa, M. (noviembre, 2005). Los desafíos de la formación docente para una educación infantil del siglo XXI: Post estructuralismo, globalización y sus implicaciones para la educación de 
la primera infancia. Conferencia en Seminario Internacional: Construyendo el futuro de los niños y niñas. Santiago.

Descouvieres, C., Redondo, J. y Rojas, K. (2005). Eficacia y eficiencia de la enseñanza media chilena desde los datos SIMCE 1994, 1998, 2001. Revista Enfoques Educacionales, 7(1), 125144

Díaz, F. (2003). Cognición situada y estrategias para el aprendizaje significativo. Revista Electrónica de Investigación Educativa, 5(2), 105-117.

Díaz, J. (2006). Identidad, adolescencia y cultura. Jóvenes secundarios en un contexto regional. Revista Mexicana de Investigación Educativa, 11(29), 431-457.

Espinoza, O., González, L. y Castillo, D. (2010). Factores que inciden en la deserción escolar y sus implicancias en sectores vulnerables: Un estudio de caso. Santiago: Universidad Academia de Humanismo Cristiano.

Espinoza, O., Castillo, D., González, L. y Loyola, J. (2012). Factores familiares asociados a la deserción escolar en Chile. Revista de Ciencias Sociales, 13(1), 136-150.

Espinoza, O., Castillo, D., González, L.E. Loyola, J. y Santa, E. (2014). Deserción escolar en Chile: un estudio de caso en relación con factores intraescolares. Educación, 17(5), 32-50.

Fraser, N. (2000). ¿De la redistribución al reconocimiento? Dilemas de la justicia en la era postsocialista. New Left Review, 1, 126-155.

Fraser, N. (2008). La justicia social en la era de la política de identidad: redistribución, reconocimiento y participación. Revista de Trabajo, 4(6), 1-19.

Gaitán, L. (2006). La nueva sociología de la infancia. Aportaciones de una mirada distinta. Política y Sociedad, 43(1), 9-26.

García-Huidobro, J. E. (2001). Educación, pobreza y fracaso escolar. Santiago: UNICEF.

Geertz, C. (2005). La interpretación de las culturas. Barcelona: Gedisa.

Gergen, K. (1992). El yo saturado: dilemas de identidad en el mundo contemporáneo. Barcelona: Paidós.

Giménez, G. (1997). Materiales para una teoría de las identidades sociales. Frontera Norte, 9(18), 9-28. Ciudad de México: CONACYT

Goicovic, I. (2002). Educación, deserción escolar e integración laboral Juvenil. Última Década, 16, 11-52. doi: 10.4067/s0718-22362002000100002

Halliday, M. (1982). El lenguaje como semiótica social. La interpretación social del lenguaje y del significado. Ciudad de México: Fondo de Cultura Económica

Íñiguez, L. (2001). De lo personal a lo social. Un recorrido conceptual. En E. Crespo (Ed.), La constitución social de la subjetividad (pp. 209-225). Madrid: Catarata.

Ivanovic, D. (2000). Característica de los alumnos asociadas a su trayectoria escolar. Revista Archivos Latinoamericanos de Nutrición, 51(1), 7-9.

Jorquera, E. y Leiva, E. (2002). Permiso para ser, permiso para hacer. La construcción de derechos de los jóvenes. Santiago: Fundación Ideas.

Julio, C. (2010). Legítimos aprendices: recuperando al sujeto en el proceso educativo. Revista Diversia, 2, 19-43.

Julio, C. (2014). Dilema identitario en el primer ciclo de educación básica municipal: Un obstáculo en la relación pedagógica que incide en la posibilidad de concluir trayectorias de aprendizaje escolar (Tesis de doctorado). Universidad Academia de Humanismo Cristiano. 
Julio, C., Conejeros-Solar, L., Rojas, C., Mohammad, M., Rubí, Y. y Cortés, Y. (2016). Desencuentro cultural en el aula: Una barrera al aprendizaje de niños y niñas en situación de pobreza. Revista Mexicana de Investigación Educativa, 21(68), 71-94.

Junta Nacional de Auxilio Escolar y Becas (2003) Los procesos de deserción enfocados desde la vulnerabilidad y la resiliencia: Un cambio de Perspectiva. Departamento Unidad de Investigación y Desarrollo Estratégico. Santiago: JUNAEB.

Kastersztein, J. (1999). Les estratégies identitaires des acteurs sociaux: approche dynamique des finalités. En Camilleri, C. et al (1999) Stratégies Identitaires. Paris: Presses Universitaires de France. Tercera Edición. Primera Edición 1990.

Lave, J. (1991). La cognición en la práctica, Barcelona: Paidós.

Lave, J. y Wenger, E. (1991). Situated learning. Legitimate peripheral participation. Nueva York, NY: Cambridge University Press. doi: 10.1017/CBO9780511815355

Lipiansky, E. M. (1999). Identité subjective et interaccion. En C. Camilleri (Ed.), Stratégies Identitaires. París: Presses Universitaires de France.

Magendzo, A. (2008). Dilemas del currículum y la pedagogía. Santiago: LOM

Marshall, G. y Correa, L. (2001). Focalización de becas del programa liceo para todos: Un estudio casocontrol. Santiago: Universidad Católica de Chile.

Maturana, H. (2007). Emociones y lenguaje en educación y política. Santiago: Comunicaciones Noreste.

Melis, F. (2001). Los niños y adolescentes fuera del sistema escolar. Análisis de la encuesta CASEN 2000. Santiago: División Social del Ministerio de Planificación Nacional

Melis, F., Díaz, R. y Palma, A. (2004). Adolescentes y jóvenes que abandonan sus estudios antes de finalizar la enseñanza media: Principales tendencias. Santiago: División Social, Ministerio de Planificación Nacional

Méndez, R. (2002). Perfil psicosocial del desertor escolar. Santiago: Adimark y Fundación Paz Ciudadana.

Mertz, C. y Uauy, C. (2002). Estudio sobre políticas y programas para la prevención de la deserción escolar en Chile. Santiago: Fundación Paz Ciudadana.

Milicic, N. (2001). Creo en ti: La construcción de la autoestima en el contexto escolar. Santiago: LOM.

Milicic, N. (2012). A ser feliz también se aprende: Guía para la educación del niño de 2 a 6 años. Santiago: Random House Mondadori.

Morin, E. (1999). Los siete saberes necesarios para la educación del futuro. París: Unesco.

Morin, E. (2002). La cabeza bien puesta. Repensar la reforma. Buenos Aires: Nueva Visión.

Morin, E., Roger, E. y Motta, R. (2003). Educar en la era planetaria. Madrid: Gedisa

OCDE (2014). Society at a glance 2014. París: OECD.

O’Brien, T. y Guiney, D. (2003). Atención a la diversidad en la enseñanza y el aprendizaje. Principios y Práctica. Madrid: Alianza Ensayo

Peralta, M. V. (enero, 2008). Articulación entre educación parvularia y básica: principios, sugerencias y estrategias. Ponencia presentada en el seminario-taller Articulación entre educación inicial y primer ciclo básico: desafíos y proyecciones. Universidad Central.

Pérez-Taylor, R. (2002). Antropología y complejidad. Barcelona: Gedisa.

Piaget, J. (2001). Inteligencia y afectividad. Buenos Aires: AIQUE. 
Raczynski, D. (2002). Procesos de deserción en la enseñanza media. Factores expulsores y protectores. Santiago: Asesorías para el Desarrollo e Instituto Nacional de la Juventud.

Restrepo, E. (2007). Identidades: Planteamientos teóricos y sugerencias metodológicas para su estudio. Revista de Antropología, 5, 24-35.

Richards, C. (2009). Proceso de deserción escolar ¿Por qué creo que me fui de la escuela? En VVAA, Experiencias educativas de segunda oportunidad. Lecciones desde la práctica innovadora en América Latina. Santiago de Chile: ORELAC/UNESCO y OEI.

Rogoff, B. (1993). Aprendices del pensamiento. Desarrollo cognitivo en el contexto social. Buenos Aires: Paidós.

Rogoff, B. (1997). Los tres planos de la actividad sociocultural: Apropiación participativa, participación guiada y aprendizaje. En H. Wertch, J. Del Río y D. Álvarez (Eds.), La mente sociocultural. Aproximaciones teóricas y aplicadas (pp. 56-87). Madrid: Fundación Infancia y Aprendizaje.

Rogoff, B. y Gutiérrez, K. (2003). Cultural ways of learning: Individual traits or repertoires of practice. Educational Researcher, 32(5), 19-25. doi: 10.3102/0013189X032005019

Román, M. (2009). Abandono y deserción escolar: Duras evidencias de la incapacidad de retención de los sistemas y de su porfiada inequidad. REICE. Revista Iberoamericana sobre Calidad, Eficacia y Cambio en Educación, 7(4), 3 - 9.

Santos, H. (2009). Dinámica de la deserción escolar en Chile. Santiago: Universidad Diego Portales.

Sapelli, C. y Torche, A. (2004). Deserción escolar y trabajo juvenil: ¿Dos caras de una misma decisión? Cuadernos de Economía, 41, 173-198. doi: 10.4067/So717-68212004012300001

Schkolnik, M. (2003). Trabajo infantil y juvenil y Educación. Chile: Ministerio del Trabajo.

Tezanos, A. (1983). Notas para una reflexión crítica sobre pedagogía. En G. Hoyos (Ed.), Sujeto como objeto de las ciencias sociales. Bogotá: CIUP.

Tezanos, A. (2005) La Torre de Babel: Los equívocos acerca de los sentidos de la educación. Ponencia en el encuentro: Los sentidos de la Educación y la Cultura. Cultivar la Humanidad. Organizado por UNESCO, Ministerio de Educación de Chile y Consejo de la Cultura y las Artes. Santiago de Chile: UNESCO.

Tezanos, A. (2007). Formación de profesores: una reflexión y una propuesta, Pensamiento Educativo, 41(2), 57-75.

Tezanos, A. (2012). ¿Identidad y/o tradición docente? Apuntes para una discusión. Perspectiva Educacional, 51(1), 1-28.

Toboada-Leonetti, I. (1999). Strategies identitaries et minorités: le point de vue du sociologue. En C. Camilleri (Ed.), Stratégies identitaires (pp. 97-123). París: Presses Universitaires de France.

UNICEF. (2006). Convención sobre los derechos del niño. Madrid: UNICEF

Wenger, E. (2001). Comunidades de práctica. Aprendizaje, significado e identidad. Barcelona: Paidós.

Ysern, J. L. (2006) Comunicación y Desarrollo en la experiencia de Radio Estrella del Mar. Primer Congreso Chileno de Estudiantes de Postgrado en Comunicación. $2^{\circ}$.Congreso de Estudiantes Escuela de Graduados Facultad de Filosofía y Humanidades. Universidad Austral de Chile - Valdivia 13, 14,15 Diciembre 2006. 


\section{Breve CV de la autora}

\section{Cristina Julio Maturana}

Tiene 31 años de experiencia profesional, es Dra. en Educación, Licenciada en Ciencias Sociales y Ética Social y Profesora de Educación Diferencial/Especial. Durante los '80 se desempeñó en aulas de escuelas especiales y en gestión social en las comunas de San Miguel, Punta Arenas y Ancud. En los '90 en gestión pública (MINSAL, FOSIS, MINEDUC) en las regiones de O`Higgins y Valparaíso. Entre el 2002 y el 2015 se desempeñó como académica en la PUCV. Actualmente es Presidenta de la Cooperativa para la Acción Pedagógica, Di-versos; consultora independiente y docente de la carrera de Educación Especial en la UPLA. Cuenta con publicaciones especializadas en revistas y congresos del área de la inclusión educativa y justicia social. ORCID ID: 0000-00017881-4248. Email: cristina.julio.m@gmail.com 